\title{
REAL- TIME ATTENDANCE SYSTEM USING FACE RECOGNITION TECHNIQUE
}

\author{
Debadrita Ghosh
}

\begin{abstract}
With increasing technologies and scientific knowledge, today's world has resulted in a great change in almost all aspects. Technical facilities, machine learning, algorithms and other aspects are playing a huge role in almost every part of the world. Taking this into consideration, this research was developed by us, which includes face recognition, face detection and feature extraction. This research is based on real time face recognition for attendance, as it may help in huge number of institutions and other sectors. Here no physical involvement of invigilator is required. The system will totally conduct the processes based on better internet connection and better illumination. An extra feature has been added which includes the details of the pupil to be emailed to their guardian. It's undertaking is done with Python 3.7.6, OpenCV 3.4. and Anaconda Navigator(Anaconda3). The proposed arrangement is tried for different light intensities and conditions.
\end{abstract}

Keywords- Face Recognition, Feature Selection, Feature Extraction methods, Face Detection, Database, Attendance, Attendance Report

\section{INTRODUCTION}

Face Recognition is a technology which works on matching the human faces from a video frame, or digital platform, against a database of faces, which is currently being used in ID verification process, biometrics, smartphones as well as robotics. Face Recognition is now widely accepted as a research topic across the globe. With the vast advancements in face recognition, more research should be done to increase the efficiency and accuracy of the method that is produced. [14] Face Recognition is a pattern recognition problem, where the face represented is subjected to varying intensities, and other factors, needs to be identified based on acquired images. [11] The objective of face recognition is, from the actual image, to find a series of same face in a set of training images in a database. This process is carried out in realtime, unlike all biometric facial recognition software providers. Since, student plays an important role in institutions and universities, it becomes very important to keep track of those students by attendance method. There are mainly two types of attendance systems:
A. Manual Attendance System.
B. Automated Attendance System.

[27] This research mainly focuses on the implementation of real time attendance system as well as sending the attendance report to the respected guardians to help them track the number of classes attended by their children, which will further be implemented in institutions, organizations as well as schools to reduce the time invested while taking the attendance by ordinary procedures.

\section{PROPOSED AlgORITHM}

The real time face recognition automated attendance system was implemented to record the attendance more efficiently and accurately. The system that we have proposed will update the attendance automatically once the face of the student is matched with the training dataset.

The proposed system will grant the permission to the student of entering the class if his/her face matches with the dataset. If any student tries to enter the wrong class, then the system will automatically identify and update the user. [8] Face recognition is developed by extracting the image using webcam under high illumination, detecting the face, and then comparing the detected face with the training database and then marking the attendance. The attendance will then get marked in a spreadsheet.

The system is mainly comprising of two main parts:

I. Development of Face Recognition System.

II. Development of Attendance System.

The basic pipeline used for face recognition through machine learning includes finding an image in a face, analysing facial features and comparing against the known faces and making a prediction and generating a detailed attendance sheet which can be mailed manually. 


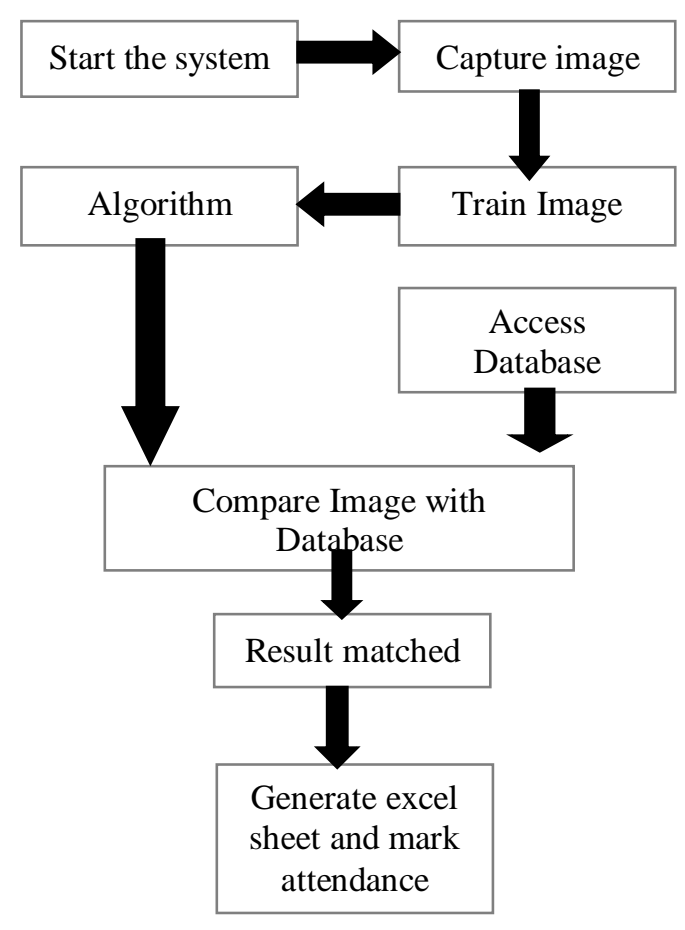

Fig. 1. Proposed System Block Diagram

\section{A. FEATURE EXTRACTION METHODS:}

Feature Extraction is the most vital stage in pattern recognition and data mining. In this stage, the meaningful feature subset is extracted from original data by applying certain rules. For reliable recognition, it is desirable to extract appropriate features space, since all the extracted features may not contribute to the classification positively. Feature extraction is the application of extracting algorithm on digital images to reduce redundancy and irrelevancy present in the image. The main goals of feature extraction are to reduce the time of machine training and complexity of space, in order to achieve a dimension reduction. Feature extraction algorithms transform an input data into the set of features, meanwhile select features containing the most relevant information from the original data. Feature extraction maintain acceptable classification accuracy by reducing maximum number of irrelevant features. It has great importance in data analysis, pattern classification, biometrics, computer vision, multimedia information retrieval, remote sensing, machine learning, medical data processing, and data mining applications. There are two common approaches to extract facial features: Geometric features and Appearance based methods.

I. Geometric Feature Based Methods: The feature-based or analytic approach computes a set of geometrical [6] face features of eyes, a mouth, and a nose. In this representation, outline of the face and positions of the different facial features form a feature vector. This includes: mouth, eyes, eyebrows and nose.

II. Holistic Based Methods: In Holistic or Appearance-based methods, the global properties of the human face pattern are considered. Unlike in feature based, the whole face region is recognized without using few points from different regions of the face.

\section{B. SYSTEM STRATEGY:}

We are completely user driven. Gray image conversion technique and HaarCascade classifiers have been used to extract the features from the captured image.

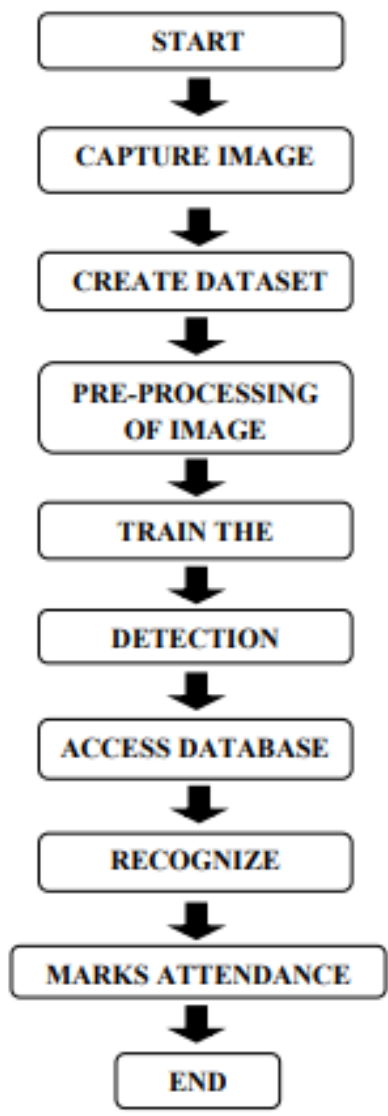

Fig. 2. Block Diagram of System Strategy.

\section{CREATE DATASET:}

The dataset includes many number of images of the indicated person, for the process of recognition. It is stored in a folder named 'DATASET'. 


\section{PRE-PROCESSING OF IMAGE:}

We have used gray image conversion technique which converts input image converts into grey scale image, for preprocessing the image for better quality.

\section{TRAIN THE DATASET:}

When algorithm is asked to recognize some unknown face, it uses training set to make the recognition. It will use the same id which was used for capturing faces, for the successful training. And the trained sheet will be successfully saved in a folder named trainer, with the extension trainer.yml.

\section{FACE DETECTION:}

Here, the human face is detected in digital form using the function,

detector $=\mathrm{cv}$ 2.CascadeClassifier("haarcascade_frontalface_de fault.xml"); 'frontalface' is used since we are using the front face of the students for automated attendance system, and haarcascade classifier is required in OpenCV for extracting the facial features of the image captured.

\section{ACCESS DATABASE:}

Database will be required to compare the face images. Previously made dataset will be accessed now.

\section{RECOGNIZE:}

When algorithm is asked to recognize some unknown face, it uses training set to make the recognition. Here, LBPH method is used.

In our proposed system face is recognized using the following function: recognizer $=$ cv2.face. LBPHFaceRecognizer_create (); Recognizer. Read('trainer/trainer.yml'); This will recognize the face using LBPH algorithm and by using the trainer.yml file, to find the exact match of faces.

\section{MARKS THE ATTENDANCE:}

If the face which was trained is matched with the face being recognized, then the system on clicking the button 'ATTENDANCE SHEET' will automatically create the attendance sheet with the current date and the name of the candidate.

\section{MAIL THE ATTENDANCE:}

After marking the attendance, the attendance sheet can be mailed to the guardians, while selecting the mail ids and the attendance sheet manually.
III. EXPERIMENT AND RESULT

\section{FLOW CHART DIAGRAM:}

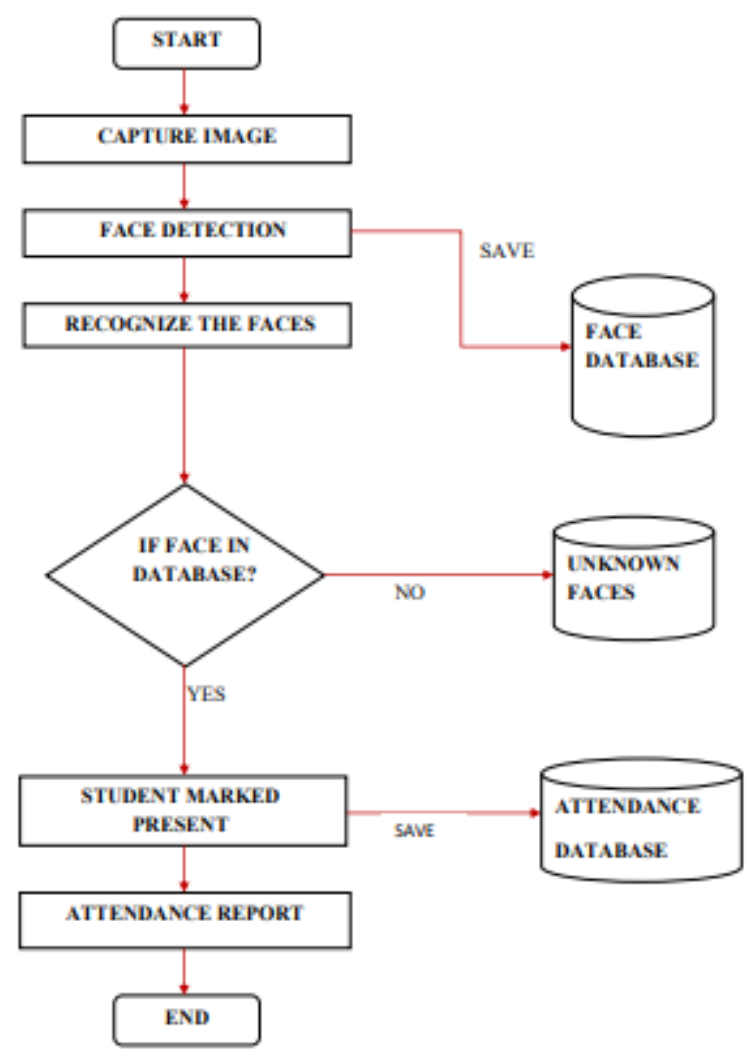

2. ENTITY-RELATIONSHIP DIAGRAM:

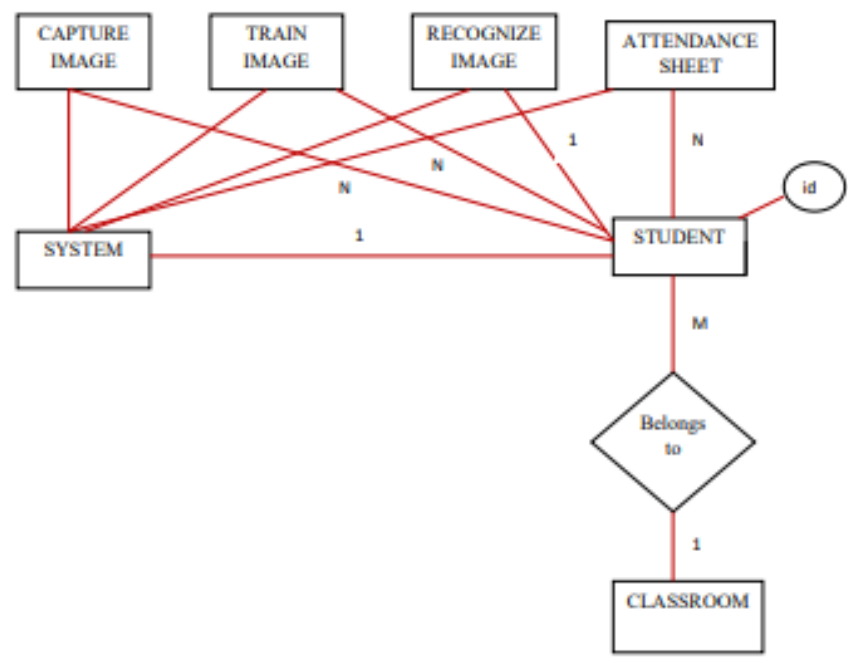




\section{EXPERIMENTAL RESULTS:}

Table -1 Experiment Result

\begin{tabular}{|c|c|c|c|}
\hline TEST DATA & $\begin{array}{c}\text { EXPECTED } \\
\text { RESULTS }\end{array}$ & $\begin{array}{c}\text { OBSERVED } \\
\text { RESULTS }\end{array}$ & $\begin{array}{c}\text { EXECUTED } \\
\text { (TRUE/FALSE) }\end{array}$ \\
\hline $\begin{array}{c}\text { cv2.VideoCapture } \\
(0)\end{array}$ & $\begin{array}{c}\text { Connects } \\
\text { with the } \\
\text { installed } \\
\text { camera and } \\
\text { starts } \\
\text { playing. }\end{array}$ & $\begin{array}{c}\text { Camera } \\
\text { started. }\end{array}$ & True \\
\hline $\begin{array}{c}\text { cv2.Cascade } \\
\text { Classifier }\end{array}$ & $\begin{array}{c}\text { Loads the } \\
\text { haarcascade } \\
\text { classifier. }\end{array}$ & $\begin{array}{c}\text { Gets ready } \\
\text { for } \\
\text { extraction. }\end{array}$ & True \\
\hline ExtractFace() & $\begin{array}{c}\text { Loads the } \\
\text { LBPH face } \\
\text { extracting } \\
\text { framework. }\end{array}$ & $\begin{array}{c}\text { Face } \\
\text { extracted. }\end{array}$ & True \\
\hline Learn() & $\begin{array}{c}\text { Starts the } \\
\text { LBPH } \\
\text { algorithm }\end{array}$ & $\begin{array}{c}\text { Updates the } \\
\text { trainer.xml } \\
\text { file. }\end{array}$ & True \\
& $\begin{array}{c}\text { It compares } \\
\text { the input } \\
\text { face with } \\
\text { the saved } \\
\text { images. }\end{array}$ & Recognizes. & True \\
\hline Recognize() & & \\
\hline
\end{tabular}

This contains the results that we achieved throughout the course of using this system. Results Achieved from initiation through conclusion of developing this system the following results has been achieved.

They are as follows:

- The system is market ready for commercial use.

- The system has the capacity to carry up to a thousand faces to recognize.

- The system can serve as much people as they want within an organization.

\section{CONCLUSION}

In the system automatic attendance have been implemented. By using the techniques proved time saving and highsecurity. It also identifies an unknown person too. The future extraction is to increase the rate of recognition techniques. This system has only developed face recognizes up-to 30 degree's angle should be improved further. The class room with a poor lighting may effect image with lack of clarity system performance degrades indirectly. Among all biometric techniques, face recognition approach possesses one of the great advantage, which is its user-friendliness and gives better accuracy than other methods for implementing system.

\section{REFERENCE}

[I] Paul Sandeep and Singh Lotika, 2015, "A review on advances in deep learning”, DOI10.1109/WCI.2015.7495514

[II] Girshick Ross, 2015, "Fast R-CNN", DOI10.1109/ICCV.2015.169

[III] Basheer M. K.P. and Raghu C. 2012, "Fingerprint Attendance System for Classroom Needs", DOI10.1109/INDCON.2012.6420657

[IV] Singha Manminder, Dr. Arorab A. S. , "Varying Illumination and Pose Conditions in Face Recognition", CSE Department SLIET, Sangrur, 148106, India b Professor, EIE Department, SLIET, Sangrur, 148106, India.

[V] Open source computer vision library.[Online] Available: https://opencv.org/

[VI] Zhao XueMei and Wei ChengBing, 2017, “A RealTime Face Recognition System Based On Improved LBPH Algorithm", DOI10.1109/SIPPROCESS.2017.8124508

[VII] Issuu.com

[VIII] Yael Moses, Yael Adini, Shimon Ullman. 1994 "Chapter 33 Face recognition: The problem of compensating for changes in illumination direction" , Springer Science and Business Media LLC.

[IX] link.springer.com

[X] Ghazal Bargshady, Xujuan Zhou, Ravinesh C. Deo, Jeffrey Soar, Frank Whittaker, Hua Wang. 2020, "Ensemble neural network approach detecting pain intensity from facial expressions" , Artificial Intelligence in Medicine

[XI] Ayan Seal, Debotosh Bhattacharjee, Mita Nasipuri, Dipak Kumar Basu. 2018, "chapter 78 Thermal Human Face Recognition for Biometric Security System" , IGI Global

[XII] Manasi Jeste, Paresh Gokhale, Shrawani Tare, Yutika Chougule, Archana Chaudhari. 2020, "Twopoint security system for doors/lockers using Machine learning and Internet Of Things" , 2020 Fourth International Conference on Inventive Systems and Control (ICISC)

[XIII] M. Gopila, D. Prasad. 2020, "Machine learning classifier model for attendance management system" , 2020 Fourth International Conference on I-SMAC (IoT in Social, Mobile, Analytics and Cloud) (ISMAC) 